International Journal of Pure and Applied Mathematics

Volume 103 No. 4 2015, 635-652

ISSN: $1311-8080$ (printed version); ISSN: 1314-3395 (on-line version)

url: http://www.ijpam.eu

doi: http://dx.doi.org/10.12732/ijpam.v103i4.4

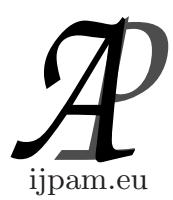

\title{
FREE FLOW OF A JEFFREY FLUID BETWEEN TWO LONG VERTICAL THIN PLATES
}

\author{
A.N.S. Srinivas ${ }^{1}$, S. Sreenadh ${ }^{2}$, B. Govindarajulu ${ }^{2}$, R. Hemadri Reddy ${ }^{1}$ \\ ${ }^{1,4}$ School of Advanced Sciences \\ VIT University \\ Vellore, Tamil Nadu, 632 014, INDIA \\ ${ }^{2,3}$ Department of Mathematics \\ Sri Venkateswara University \\ Tirupati, 517 502, INIDA
}

\begin{abstract}
Free convection flow of a Jeffrey fluid between two long vertical plates is investigated. The vertical plates are moving with some velocity but in opposite directions. The coupled governing equations, of which one of them is nonlinear, are solved by the linearization technique. It is observed that out of all the physical parameters, the effect of non-Newtonian Jeffrey parameter is the strongest on the velocity and temperature of the fluid. The numerical results obtained reveal many interesting behavior of Grashof number G, Prandtl numbers $\mathrm{P}$, the Eckert number E and the plate velocity V. Furthermore, some deductions are carried out to compare the present results with those previously established.
\end{abstract}

Key Words: free convection, heat transfer, vertical plates, Jeffrey parameter

Received: March 17, 2015

(c) 2015 Academic Publications, Ltd. url: www.acadpubl.eu

${ }^{\S}$ Correspondence author 


\section{Introduction}

Considerable work has been performed on the purely forced convection flow of non-Newtonian fluids. In contrast, relatively little work has been reported on the effects of free convection on non-Newtonian fluids. Over the past few years, considerable research effort has been devoted to the study of heat transfer induced by buoyancy effects in a porous medium saturated with fluids. Interest in this convective flow phenomenon has been motivated by diverse engineering problems, such as underground heat exchangers for energy storage, recovery and temperature controlled reactors, electronic systems cooling, petroleum reservoirs, groundwater hydrology, coal combustors, grain storage, and fiber, granular insulation and geothermal energy extraction. In studies of free and forced convection heat transfer in fluids flowing between long vertical plates, the Grashof number G plays a significant role in affecting the fluid flow and heat transfer characteristics. Suriano et al. [1] have stressed the importance of small Grashof numbers in the study of the laminar free convection heat transfer.

The flow of a viscous fluid past an infinite vertical porous plate with convective acceleration is studied by Yamamoto and Yoshida [2]. Vajravelu [9] studied the free convection heat transfer between two long, vertical, thin plates moving in opposite directions. Rajasekhara et al. [4] investigated the Couette flow over a naturally permeable bed. It is found that the flux is greatly enhanced due to the introduction of permeable bed. Rudraiah and Veerabhadraiah [5] studied the temperature distribution in a steady plane Couette flow past a permeable bed in the presence of buoyancy force. A mathematical model for the study of flow between two permeable beds is given by Sreenadh and Arunachalam [6]. The influence of permeability on the velocity distributions in the porous and non-porous regions are discussed in detail. Rajagopal and Gupta [7] obtained an exact solution for the flow of a non-Newtonian fluid past an infinite porous plate. Ogulu and Amos [8] have investigated the free convective flow of a non-Newtonian fluid past a vertical porous plate. Prathap kumar et al. [9] considered mixed convection in a vertical channel containing a composite porous medium. The influence of heat transfer on the peristaltic transport of a Jeffrey fluid in a vertical porous stratum is studied by Vajravelu et al [10]. Umavathi and Shekar [11] studied the unsteady mixed convective flow and heat transfer in a vertical corrugated channel with composite porous media.

Free convection flow and heat transfer studies of a viscous fluid confined between two long, thin vertical plates finds important applications in industrial engineering. Such a study is useful in assessing the effect of the motion of a broad belt on the flow field. Motivated by this, we propose to study free con- 
vection effects on the flow and heat transfer of a Jeffrey fluid confined between two long, parallel, vertical plates moving with equal velocities but in opposite directions. In this study, quite a few interesting features of the flow and heat transfer characteristics have been discussed. Some deductions are made and the results are discussed through graphs.

\section{Formulation and Solution of the Problem}

Consider a long vertical belt or two long, thin vertical plates are as shown in Figure 1. Assume it to be broad enough to contain within it an infinite amount of a Jeffrey fluid. Let $\mathrm{X}$ - axis is taken along the plates in the upward direction and $\mathrm{Y}$-axis along the distance between the plates. When the flow takes place in the XY-plane, and as the plates are very long and broad compared with the distance between them, the fluid flow may be assumed to be fully developed. The parallel vertical plates are moving with common velocity $\mathrm{V}^{*}$ in opposite directions. Let the end conditions be neglected. We may take the velocity fluid as $(\mathrm{U}(\mathrm{y}), 0,0)$ and the temperature field as $(\mathrm{T}(\mathrm{y}), 0,0)$.

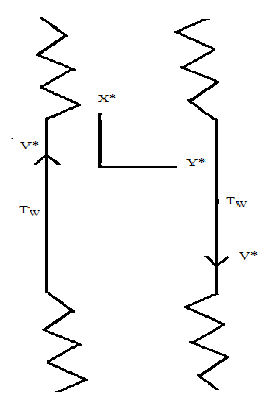

Figure 1: Physical Model

The governing equations of momentum and energy become

$$
\begin{aligned}
& \frac{d^{2} U}{d Y^{2}}-\frac{1+\lambda_{1}}{\mu}\left(\frac{d p}{d x}\right)-\frac{\rho\left(1+\lambda_{1}\right)}{\mu} g_{x}=0, \\
& \frac{d p}{d Y}=0, \\
& \frac{d^{2} T}{d Y^{2}}+\frac{\mu}{\left(1+\lambda_{1}\right) K_{0}}\left(\frac{d U}{d Y}\right)^{2}=0,
\end{aligned}
$$

where $\rho$ is the density, $\mathrm{p}$ the pressure, $\mu$ the coefficient of viscosity, $K_{0}$ the thermal conductivity, $g_{x}$ acceleration due to gravitation and $\lambda_{1}$ is the Jeffrey 
parameter. On using the well known Boussinesq approximation, namely

$$
\frac{\rho-\rho_{s}}{\rho}=-\beta\left(T-T_{s}\right)
$$

the density in equation (1) and making simplification (see Vajravelu [9], [10]), we rewrite equations (1)-(3) as

$$
\begin{aligned}
\frac{d^{2} U}{d Y^{2}}-\frac{\left(1+\lambda_{1}\right) \rho g_{x} \beta}{\mu}\left(T-T_{s}\right) & =0 \\
\frac{d^{2} T}{d Y^{2}}+\frac{\mu}{\left(1+\lambda_{1}\right) K_{0}}\left(\frac{d U}{d Y}\right)^{2} & =0 .
\end{aligned}
$$

The relevant boundary conditions of the problem are

$$
\begin{aligned}
& U=V^{*} ; T=T_{w} \quad \text { at } \quad Y=0, \\
& U=-V^{*} ; T=T_{w} \quad \text { at } \quad Y=d .
\end{aligned}
$$

Introducing the non-dimensional variables

$$
y=\frac{Y}{d} ; \quad u=\frac{U d}{v} ; \quad V=\frac{V^{*} d}{v} ; \quad \theta=\frac{T-T_{s}}{T_{w}-T_{s}}
$$

in the equations (5), (6) and the boundary conditions (7), (8) we obtain

$$
\begin{aligned}
& \frac{d^{2} u}{d y^{2}}+\left(1+\lambda_{1}\right) G \theta=0, \\
& \frac{d^{2} \theta}{d y^{2}}+\frac{P E}{\left(1+\lambda_{1}\right)}\left(\frac{d u}{d y}\right)^{2}=0, \\
& u=V ; \theta=1 \quad \text { at } \quad y=0, \\
& u=-V ; \theta=1 \quad \text { at } \quad y=1,
\end{aligned}
$$

where

$$
G=\frac{d^{3} g_{x} \beta\left(T_{w}-T_{s}\right)}{v^{2}}, \quad E=\frac{v^{2}}{d^{2} C_{p}\left(T_{w}-T_{s}\right)}, \quad P=\frac{\mu C_{p}}{K_{0}} .
$$

In order to solve the boundary value problem (9) and (10) subject to the conditions (11) we apply the iterative method (see Vajravelu, 1978) as given below:

$$
\frac{d^{2} u_{i}}{d y^{2}}+\left(1+\lambda_{1}\right) G \theta_{i}=0
$$




$$
\begin{aligned}
& \frac{d^{2} \theta_{i}}{d y^{2}}+\frac{P E}{\left(1+\lambda_{1}\right)}\left(\frac{d u_{i-1}}{d y}\right)^{2}=0 \\
& u_{i}=V ; \theta=1 \quad \text { at } \quad y=0 \\
& u_{i}=-V ; \theta=1 \quad \text { at } \quad y=1
\end{aligned}
$$

Initially $u_{0}=0$ for $i \geq 1$, then we write the solution as

$$
u_{1}=\frac{B}{2} y^{2}-\left(\frac{B+4 V}{2}\right) y+V
$$

where

$$
\begin{aligned}
& B=-\left(1+\lambda_{1}\right) G, \\
& \theta_{1}=1,
\end{aligned}
$$

$$
\begin{aligned}
u_{2}= & V-2 V y \\
& +\frac{P E}{1+\lambda_{1}}\left\{\frac{G^{3}\left(1+\lambda_{1}\right)^{3}}{360} y^{6}-\frac{G^{2}\left(1+\lambda_{1}\right)^{2}}{60}\left(\frac{G\left(1+\lambda_{1}\right)}{2}-2 V\right) y^{5}\right. \\
& +\frac{G\left(1+\lambda_{1}\right)}{24}\left(\frac{G\left(1+\lambda_{1}\right)}{2}-2 V\right)^{2} y^{4} \\
& +\frac{G\left(1+\lambda_{1}\right)}{6}\left(-\frac{G^{2}\left(1+\lambda_{1}\right)^{2}}{12}+\frac{G\left(1+\lambda_{1}\right)}{3}\left(\frac{G\left(1+\lambda_{1}\right)}{2}-2 V\right)\right. \\
& \left.-\frac{1}{2}\left(\frac{G\left(1+\lambda_{1}\right)}{2}-2 V\right)^{2}\right) y^{3} \\
& -\frac{G\left(1+\lambda_{1}\right)}{2} y^{2}-\left[\frac{G^{3}\left(1+\lambda_{1}\right)^{3}}{360}-\frac{G^{2}\left(1+\lambda_{1}\right)^{2}}{60}\left(\frac{G\left(1+\lambda_{1}\right)}{2}-2 V\right)\right. \\
& +\frac{G\left(1+\lambda_{1}\right)}{24}\left(\frac{G\left(1+\lambda_{1}\right)}{2}-2 V\right)^{2}-\frac{G\left(1+\lambda_{1}\right)}{2} \\
& +\frac{G\left(1+\lambda_{1}\right)}{6}\left(-\frac{G^{2}\left(1+\lambda_{1}\right)^{2}}{12}+\frac{G\left(1+\lambda_{1}\right)}{3}\left(\frac{G\left(1+\lambda_{1}\right)}{2}-2 V\right)\right. \\
& \left.\left.-\frac{1}{2}\left(\frac{G\left(1+\lambda_{1}\right)}{2}-2 V\right)^{2}\right) y\right\},
\end{aligned}
$$




$$
\begin{aligned}
\theta_{2}= & -\frac{P E}{1+\lambda_{1}}\left\{\frac{G^{2}\left(1+\lambda_{1}\right)^{2}}{12} y^{4}-\frac{G\left(1+\lambda_{1}\right)}{3}\left(\frac{G\left(1+\lambda_{1}\right)}{2}-2 V\right) y^{3}\right. \\
& +\frac{1}{2}\left(\frac{G\left(1+\lambda_{1}\right)}{2}-2 V\right)^{2} y^{2}-\frac{G^{2}\left(1+\lambda_{1}\right)^{2}}{12} y \\
& -\frac{G\left(1+\lambda_{1}\right)}{3}\left(\frac{G\left(1+\lambda_{1}\right)}{2}-2 V\right) y \\
& \left.+\frac{1}{2}\left(\frac{G\left(1+\lambda_{1}\right)}{2}-2 V\right)^{2} y\right\} \\
u_{3}= & \frac{m_{2} T_{1}}{182} y^{14}+\frac{m_{2} T_{2}}{156} y^{13}+\frac{m_{2} T_{3}}{132} y^{12}+\frac{m_{2} T_{4}}{110} y^{11}+\frac{m_{2} T_{5}}{90} y^{10}+\frac{m_{2} T_{6}}{72} y^{9} \\
+ & \frac{m_{2} T_{7}}{56} y^{8}+\frac{m_{2} T_{8}}{42} y^{7}+\frac{m_{2} T_{9}}{30} y^{6}+\frac{m_{2} T_{10}}{20} y^{5}+\frac{m_{2} T_{11}}{12} y^{4} \\
+ & \frac{m_{2} T_{12}}{6} y^{3}+\frac{m_{2} T_{13}}{2} y^{2}+C_{5} y+C_{6}, \\
& \theta_{3}=T_{1} y^{12}+T_{2} y^{11}+T_{3} y^{10}+T_{4} y^{9}+T_{5} y^{8}+T_{6} y^{7}+T_{7} y^{6}+T_{8} y^{5} \\
& +T_{9} y^{4}+T_{10} y^{3}+T_{11} y^{2}+T_{12} y^{1}+T_{13} .
\end{aligned}
$$

\section{Shear Stress}

The shear stress in dimensionless form is given by

$$
\begin{aligned}
\tau= & \left(\frac{d u}{d y}\right)=\frac{14 m_{2} T_{1}}{182} y^{13}+\frac{13 m_{2} T_{2}}{156} y^{12}+\frac{12 m_{2} T_{3}}{132} y^{11}+\frac{11 m_{2} T_{4}}{110} y^{10} \\
& +\frac{10 m_{2} T_{5}}{90} y^{9}+\frac{9 m_{2} T_{6}}{72} y^{8}+\frac{8 m_{2} T_{7}}{56} y^{7}+\frac{7 m_{2} T_{8}}{42} y^{6}+\frac{6 m_{2} T_{9}}{30} y^{5} \\
& +\frac{5 m_{2} T_{10}}{20} y^{4}+\frac{4 m_{2} T_{11}}{12} y^{3}+\frac{3 m_{2} T_{12}}{6} y^{2}+\frac{2 m_{2} T_{13}}{2} y+C_{5}
\end{aligned}
$$

The shear stresses at the plates are given by

$$
\begin{aligned}
& \tau_{1}=\left(\frac{d u}{d y}\right)_{y=0}=C_{5} \\
& \tau_{2}=\left(\frac{d u}{d y}\right)_{y=1}=\frac{14 m_{2} T_{1}}{182}+\frac{13 m_{2} T_{2}}{156}+\frac{12 m_{2} T_{3}}{132}+\frac{11 m_{2} T_{4}}{110}+\frac{10 m_{2} T_{5}}{90}
\end{aligned}
$$




$$
\begin{aligned}
& +\frac{9 m_{2} T_{6}}{72}+\frac{8 m_{2} T_{7}}{56}+\frac{7 m_{2} T_{8}}{42}+\frac{6 m_{2} T_{9}}{30}+\frac{5 m_{2} T_{10}}{20} \\
& +\frac{4 m_{2} T_{11}}{12}+\frac{3 m_{2} T_{12}}{6}+\frac{2 m_{2} T_{13}}{2}+C_{5} .
\end{aligned}
$$

\section{Rate of Heat Transfer}

The Nusselt number in dimensionless form is defined by

$$
\begin{aligned}
N u= & \frac{d T}{d y}=12 T_{1} y^{11}+11 T_{2} y^{10}+10 T_{3} y^{9}+9 T_{4} y^{8}+8 T_{5} y^{7}+7 T_{6} y^{6} \\
& +6 T_{7} y^{5}+5 T_{8} y^{4}+4 T_{9} y^{3}+3 T_{10} y^{2}+2 T_{11} y+T_{12} .
\end{aligned}
$$

The Nusselt numbers at the plates $\mathrm{y}=0$ and $\mathrm{y}=1$ are given by

$$
\begin{aligned}
N u_{1}= & \left(\frac{d T}{d y}\right)_{y=0}=T_{12} \\
N u_{2}= & \left(\frac{d T}{d y}\right)_{y=1}=12 T_{1}+11 T_{2}+10 T_{3}+9 T_{4}+8 T_{5}+7 T_{6}+6 T_{7}+5 T_{8} \\
& +4 T_{9}+3 T_{10}+2 T_{11}+T_{12},
\end{aligned}
$$

where

$$
\begin{gathered}
m_{1}=\frac{-P E}{1+\lambda_{1}}, \quad m_{2}=B=-\left(1+\lambda_{1}\right) G, \quad A=6 P_{1}, \\
B=5 P_{2}, \quad C=4 P_{3}, \quad D=3 P_{4}, \quad E=2 P_{5}, \quad F=P_{6}, \\
P_{1}=\frac{m_{2} a}{360} ; P_{2}=\frac{m_{2} b}{120} ; P_{3}=\frac{m_{2} c}{24} ; P_{4}=\frac{-m_{2}(a+2 b+6 c)}{72} ; P_{5}=\frac{m_{2}}{2} ; \\
P_{6}=-2 V-m_{2}\left[\frac{a}{360}+\frac{b}{120}+\frac{c}{24}-\left(\frac{a+2 b+6 c}{72}\right)-\frac{1}{2}\right] ; P_{7}=V \\
a=-m_{1} m_{2}^{2} ; b=m_{1}\left(m_{2}^{2}+4 m_{2} V\right) ; c=\frac{-P E}{1+\lambda_{1}}\left(\frac{m_{2}+4 V}{2}\right) \\
T_{1}=\frac{m_{1} A^{2}}{132} ; \quad T_{2}=\frac{m_{1} A B}{110} ; \quad T_{3}=\frac{m_{1}\left(2 A C+B^{2}\right)}{90} \\
T_{4}=\frac{m_{1}(2 A D+2 B C)}{72} ; \quad T_{5}=\frac{m_{1}\left(2 A E+2 B D+C^{2}\right)}{56} ;
\end{gathered}
$$




$$
\begin{gathered}
T_{6}=\frac{m_{1}(2 A F+2 B E+2 C D)}{42} ; T_{7}=\frac{m_{1}\left(2 B F+2 C F+D^{2}\right)}{30} ; \\
T_{8}=\frac{m_{1}(2 C F+2 D E)}{20} ; T_{9}=\frac{m_{1}\left(2 D F+E^{2}\right)}{12} ; T_{10}=\frac{m_{1} E F}{3} \\
T_{11}=\frac{m_{1} F^{2}}{2} ; \quad T_{12}=C_{3} ; \quad T_{13}=1 .
\end{gathered}
$$

Further iterations can be carried out to obtain $u_{i}, \theta_{i}$ for $i \geq 4$. It has been found that $u_{2} ; u_{3}$ and $\theta_{2} ; \theta_{3}$ are in good agreement up to the eight decimal places. We shall call $u_{3}$ as $u$ and $\theta_{3}$ as $\theta$

\section{Discussion of the Results}

The numerical results are obtained for the velocity $\mathrm{u}$ and the temperature $\theta$ for the flow of a Jeffrey fluid between thin plates using iteration method. Fluids with high viscosity will have low Grashof number G, as their Prandtl numbers are large. The smallness of the Grashof number $\mathrm{G}$ may be due to smallness of the temperature differences. Therefore, the calculated numerical results are expected to be valid for fluids.

Figure 3 shows the behavior of flow of air $\mathrm{P}=0.71$ for different values of $\mathrm{E}$, $\mathrm{P}, \mathrm{V}$, and $\lambda_{1}$. We notice that an increase in $\mathrm{G}$ results an increase in the fluid velocity near the plate $\mathrm{y}=0$ and opposite behavior is noticed near the plate $\mathrm{y}=1$. An increase in E causes comparatively small enhancement in the fluid velocity. It is found that of the Jeffrery parameter $\lambda_{1}$ causes an increase in the fluid velocity. It is clear that the plate velocity $\mathrm{V}$ enhances the fluid velocity near the plate $\mathrm{y}=0$ and decreases near the other plate. All the behavior mentioned above in the case of air is true in the case of fluids with high Prandtl numbers as shown in Figure 4.

The temperature of air at $\mathrm{P}=0.71$ with variation in $\mathrm{G}, \mathrm{E}, \mathrm{V}$ and $\lambda_{1}$ is shown in figure 4. The temperatures of air in the case when the plates are moving are higher than those when the plates are at rest. The increase in the temperature becomes very important when the plates start moving faster. We notice that the increase in $\mathrm{G}$ and $\mathrm{E}$, the fluid temperature increases. The fluid temperature increases with the increase in Prandtl number as shown in Figure 5. The temperature decreases with an increase of Jeffrey parameter $\lambda_{1}$.

From the Figures 6 and 7, it is clear that the skin friction at both the plates decreases as the plate velocity increases, while it increases with the increase in any of the other parameters G,E, $\lambda_{1}$ and P. The skin friction is positive at the upward moving plate and negative at the other plate. The variation in the rate 
of heat transfer coefficient at both the plates with the variation in $\mathrm{G}, \lambda_{1}$ and $\mathrm{E}$ in the case of air and water at $20^{\circ} \mathrm{C}$ is shown in the Figure 8 and 9 . In all the cases considered, the rate of heat transfer coefficient is positive at the upward moving plate and negative at the other, the physical meaning of which is that the heat flow at either plate is from the fluid only. The effect of $\mathrm{E}$ and $\lambda_{1}$ is to increase the rate of heat transfer coefficient at the upward moving plate and to diminish it at the other, while the effect of $G$ is to diminish the rate of heat transfer coefficient at both the plates.

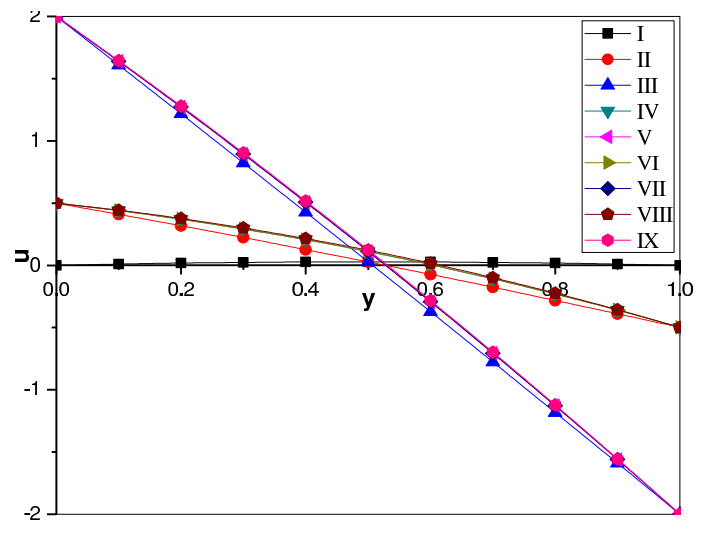

\begin{tabular}{|c|c|c|c|c|}
\hline $\mathrm{G}$ & $\mathrm{E}$ & $\mathrm{V}$ & $\lambda_{1}$ & \\
\hline 0.2 & 0.01 & 0 & 0.1 & $\mathrm{I}$ \\
\hline 0.2 & 0.01 & 0.5 & 0.1 & $\mathrm{II}$ \\
\hline 0.2 & 0.01 & 2 & 0.1 & $\mathrm{III}$ \\
\hline 0.8 & 0.01 & 0.5 & 0.1 & $\mathrm{IV}$ \\
\hline 0.8 & 0.01 & 2 & 0.1 & $\mathrm{~V}$ \\
\hline 0.8 & 0.02 & 0.5 & 0.1 & VI \\
\hline 0.8 & 0.02 & 2 & 0.1 & VII \\
\hline 0.8 & 0.02 & 0.5 & 0.2 & VIII \\
\hline 0.8 & 0.02 & 2 & 0.2 & IX \\
\hline
\end{tabular}

\begin{tabular}{|c|c|c|c|c|c|c|c|c|c|}
\hline $\mathrm{Y}$ & I & II & III & IV & V & VI & VII & VIII & IX \\
\hline 0 & 0 & 0.5 & 2 & 0.5 & 2 & 0.5 & 2 & 0.5 & 2 \\
\hline 0.1 & 0.0099 & 0.40991 & 1.61 & 0.43962 & 1.63997 & 0.43965 & 1.64033 & 0.44325 & 1.64393 \\
\hline 0.2 & 0.0176 & 0.31761 & 1.2178 & 0.37044 & 1.2711 & 0.37049 & 1.27179 & 0.37689 & 1.27819 \\
\hline 0.3 & 0.0231 & 0.22312 & 0.8233 & 0.29246 & 0.89336 & 0.29252 & 0.89431 & 0.30092 & 0.90271 \\
\hline 0.4 & 0.0264 & 0.12642 & 0.4267 & 0.20567 & 0.50672 & 0.20574 & 0.50785 & 0.21534 & 0.51745 \\
\hline 0.5 & 0.0275 & 0.02752 & 0.0278 & 0.11008 & 0.11119 & 0.11015 & 0.11237 & 0.12015 & 0.12237 \\
\hline 0.6 & 0.0264 & -0.0736 & -0.3733 & 0.00567 & -0.29327 & 0.00575 & -0.29213 & 0.01535 & -0.28253 \\
\hline 0.7 & 0.0231 & -0.1769 & -0.7767 & -0.10754 & -0.70663 & -0.10747 & -0.70566 & -0.09907 & -0.69725 \\
\hline 0.8 & 0.0176 & -0.2824 & -1.1822 & -0.22955 & -1.12889 & -0.2295 & -1.12818 & -0.2231 & -1.12177 \\
\hline 0.9 & 0.0099 & -0.3901 & -1.59 & -0.36038 & -1.56002 & -0.36035 & -1.55965 & -0.35675 & -1.55604 \\
\hline 1 & 0 & -0.5 & -2 & -0.5 & -2 & -0.5 & -2 & -0.5 & -2 \\
\hline
\end{tabular}

Figure 2: Velocity distribution for $\mathrm{P}=0.71$ 


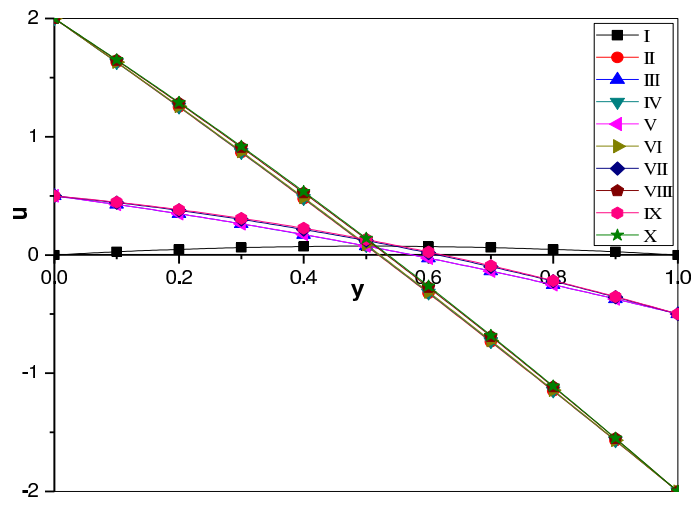

\begin{tabular}{|c|c|c|c|c|c|}
\hline $\mathrm{G}$ & $\mathrm{E}$ & $\mathrm{P}$ & $\mathrm{V}$ & $\lambda_{1}$ & \\
\hline 0.5 & 0.01 & 3 & 0 & 0.1 & $\mathrm{I}$ \\
\hline 0.5 & 0.01 & 3 & 2 & 0.1 & $\mathrm{II}$ \\
\hline 0.5 & 0.01 & 7 & 0.5 & 0.1 & $\mathrm{III}$ \\
\hline 0.5 & 0.01 & 7 & 2 & 0.1 & $\mathrm{IV}$ \\
\hline 0.5 & 0.02 & 7 & 0.5 & 0.1 & $\mathrm{~V}$ \\
\hline 0.5 & 0.02 & 7 & 2 & 0.1 & $\mathrm{VI}$ \\
\hline 0.8 & 0.01 & 7 & 0.5 & 0.1 & $\mathrm{VII}$ \\
\hline 0.8 & 0.01 & 7 & 2 & 0.1 & $\mathrm{VIII}$ \\
\hline 0.8 & 0.01 & 7 & 0.5 & 0.2 & $\mathrm{IX}$ \\
\hline 0.8 & 0.01 & 7 & 2 & 0.2 & $\mathrm{X}$ \\
\hline
\end{tabular}

\begin{tabular}{|c|c|c|c|c|c|c|c|c|c|c|}
\hline $\mathrm{Y}$ & $\mathrm{I}$ & $\mathrm{II}$ & III & IV & V & VI & VII & VIII & IX & X \\
\hline 0 & 0 & 2 & 0.5 & 2 & 0.5 & 2 & 0.5 & 2 & 0.5 & 2 \\
\hline 0.1 & 0.027 & 1.62797 & 0.42714 & 1.62927 & 0.42728 & 1.63153 & 0.44343 & 1.64681 & 0.44703 & 1.65041 \\
\hline 0.2 & 0.048 & 1.24984 & 0.34827 & 1.2523 & 0.34853 & 1.25659 & 0.37723 & 1.28366 & 0.38363 & 1.29005 \\
\hline 0.3 & 0.063 & 0.86553 & 0.26337 & 0.8689 & 0.26374 & 0.87478 & 0.3014 & 0.91022 & 0.3098 & 0.91862 \\
\hline 0.4 & 0.072 & 0.47497 & 0.17244 & 0.47892 & 0.17287 & 0.48584 & 0.21591 & 0.52628 & 0.22552 & 0.53588 \\
\hline 0.5 & 0.075 & 0.07813 & 0.07546 & 0.08229 & 0.07593 & 0.08957 & 0.12076 & 0.13168 & 0.13077 & 0.14169 \\
\hline 0.6 & 0.072 & -0.32501 & -0.0276 & -0.32104 & -0.02711 & -0.31409 & 0.01594 & -0.27363 & 0.02554 & -0.26402 \\
\hline 0.7 & 0.063 & -0.73444 & -0.1366 & -0.73104 & -0.13623 & -0.72509 & -0.09856 & -0.68962 & -0.09015 & -0.68121 \\
\hline 0.8 & 0.048 & -1.15013 & -0.2517 & -1.14764 & -0.25143 & -1.14328 & -0.22273 & -1.11618 & -0.21632 & -1.10977 \\
\hline 0.9 & 0.027 & -1.57201 & -0.3729 & -1.57069 & -0.3727 & -1.56839 & -0.35655 & -1.55308 & -0.35294 & -1.54947 \\
\hline 1 & 0 & -2 & -0.5 & -2 & -0.5 & -2 & -0.5 & -2 & -0.5 & -2 \\
\hline
\end{tabular}

Figure 3: Velocity distribution for $\mathrm{P}=7$ 


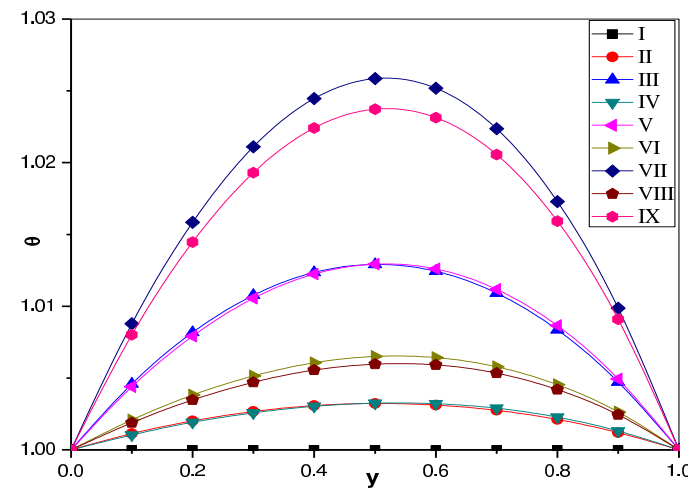

\begin{tabular}{|c|c|c|c|c|}
\hline $\mathrm{G}$ & $\mathrm{E}$ & $\mathrm{V}$ & $\lambda_{1}$ & \\
\hline 0.2 & 0.01 & 0 & 0.1 & $\mathrm{I}$ \\
\hline 0.2 & 0.01 & 1 & 0.1 & $\mathrm{II}$ \\
\hline 0.2 & 0.01 & 2 & 0.1 & $\mathrm{III}$ \\
\hline 0.8 & 0.01 & 1 & 0.1 & $\mathrm{IV}$ \\
\hline 0.8 & 0.01 & 2 & 0.1 & $\mathrm{~V}$ \\
\hline 0.8 & 0.02 & 1 & 0.1 & $\mathrm{VI}$ \\
\hline 0.8 & 0.02 & 2 & 0.1 & $\mathrm{VII}$ \\
\hline 0.8 & 0.02 & 1 & 0.2 & VIII \\
\hline 0.8 & 0.02 & 2 & 0.2 & IX \\
\hline
\end{tabular}

\begin{tabular}{|c|c|c|c|c|c|c|c|c|c|}
\hline Y & I & II & III & IV & V & VI & VII & VIII & IX \\
\hline 0 & 1 & 1 & 1 & 1 & 1 & 1 & 1 & 1 & 1 \\
\hline 0.1 & 1 & 1.00113 & 1.0046 & 1.00104 & 1.00439 & 1.00208 & 1.00878 & 1.00189 & 1.00801 \\
\hline 0.2 & 1 & 1.00202 & 1.0082 & 1.00191 & 1.00792 & 1.00381 & 1.01584 & 1.00347 & 1.01447 \\
\hline 0.3 & 1 & 1.00267 & 1.0108 & 1.00258 & 1.01055 & 1.00515 & 1.0211 & 1.00471 & 1.0193 \\
\hline 0.4 & 1 & 1.00308 & 1.0124 & 1.00303 & 1.01224 & 1.00607 & 1.02447 & 1.00555 & 1.02241 \\
\hline 0.5 & 1 & 1.00323 & 1.0129 & 1.00325 & 1.01293 & 1.00651 & 1.02586 & 1.00597 & 1.02372 \\
\hline 0.6 & 1 & 1.00312 & 1.0124 & 1.00321 & 1.0126 & 1.00643 & 1.02519 & 1.00592 & 1.02313 \\
\hline 0.7 & 1 & 1.00275 & 1.0109 & 1.0029 & 1.01118 & 1.00579 & 1.02237 & 1.00534 & 1.02056 \\
\hline 0.8 & 1 & 1.00211 & 1.0084 & 1.00227 & 1.00865 & 1.00454 & 1.01729 & 1.0042 & 1.01592 \\
\hline 0.9 & 1 & 1.0012 & 1.0047 & 1.00131 & 1.00493 & 1.00263 & 1.00987 & 1.00244 & 1.00909 \\
\hline 1 & 1 & 1 & 1 & 1 & 1 & 1 & 1 & 1 & 1 \\
\hline
\end{tabular}

Figure 4: Temperature distribution for $\mathrm{P}=0.71$ 


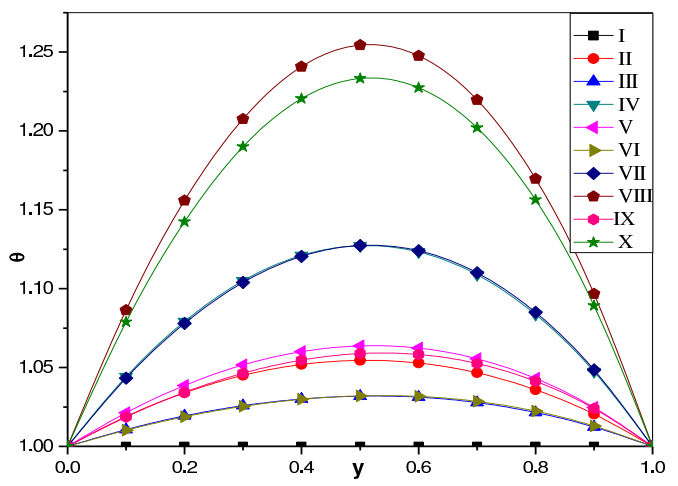

\begin{tabular}{|c|c|c|c|c|c|}
\hline $\mathrm{G}$ & $\mathrm{E}$ & $\mathrm{P}$ & $\mathrm{V}$ & $\lambda_{1}$ & \\
\hline 0.5 & 0.01 & 3 & 0 & 0.1 & $\mathrm{I}$ \\
\hline 0.5 & 0.01 & 3 & 2 & 0.1 & $\mathrm{II}$ \\
\hline 0.5 & 0.01 & 7 & 1 & 0.1 & $\mathrm{III}$ \\
\hline 0.5 & 0.01 & 7 & 2 & 0.1 & $\mathrm{IV}$ \\
\hline 0.5 & 0.02 & 7 & 1 & 0.1 & $\mathrm{~V}$ \\
\hline 0.5 & 0.01 & 7 & 2 & 0.1 & $\mathrm{VI}$ \\
\hline 0.8 & 0.01 & 7 & 1 & 0.1 & $\mathrm{VII}$ \\
\hline 0.8 & 0.02 & 7 & 2 & 0.1 & $\mathrm{VIII}$ \\
\hline 0.8 & 0.01 & 7 & 1 & 0.2 & $\mathrm{IX}$ \\
\hline 0.8 & 0.01 & 7 & 2 & 0.2 & $\mathrm{X}$ \\
\hline
\end{tabular}

\begin{tabular}{|c|c|c|c|c|c|c|c|c|c|c|}
\hline $\mathrm{Y}$ & $\mathrm{I}$ & $\mathrm{II}$ & $\mathrm{III}$ & $\mathrm{IV}$ & $\mathrm{V}$ & $\mathrm{VI}$ & $\mathrm{VII}$ & $\mathrm{VIII}$ & $\mathrm{IX}$ & $\mathrm{x}$ \\
\hline 0 & 1 & 1 & 1 & 1 & 1 & 1 & 1 & 1 & 1 & 1 \\
\hline 0.1 & 1.00003 & 1.01893 & 1.01067 & 1.04415 & 1.0213 & 1.01026 & 1.04324 & 1.08641 & 1.01864 & 1.0788 \\
\hline 0.2 & 1.00004 & 1.03397 & 1.01932 & 1.07922 & 1.0386 & 1.01879 & 1.07802 & 1.15589 & 1.03422 & 1.1424 \\
\hline 0.3 & 1.00004 & 1.045 & 1.02583 & 1.10494 & 1.0516 & 1.0254 & 1.10392 & 1.20761 & 1.04639 & 1.1899 \\
\hline 0.4 & 1.00004 & 1.0519 & 1.03007 & 1.12104 & 1.0601 & 1.02989 & 1.12051 & 1.24073 & 1.05473 & 1.2205 \\
\hline 0.5 & 1.00004 & 1.05456 & 1.0319 & 1.12724 & 1.0638 & 1.03206 & 1.12736 & 1.25438 & 1.05884 & 1.2333 \\
\hline 0.6 & 1.00004 & 1.05286 & 1.03118 & 1.12326 & 1.0623 & 1.03168 & 1.12404 & 1.24769 & 1.05829 & 1.2275 \\
\hline 0.7 & 1.00004 & 1.04667 & 1.02778 & 1.10882 & 1.0555 & 1.02852 & 1.11009 & 1.21977 & 1.05261 & 1.2021 \\
\hline 0.8 & 1.00004 & 1.03588 & 1.02155 & 1.08365 & 1.0431 & 1.02236 & 1.08507 & 1.16975 & 1.04133 & 1.1563 \\
\hline 0.9 & 1.00002 & 1.02037 & 1.01234 & 1.04747 & 1.0247 & 1.01293 & 1.04852 & 1.09678 & 1.02397 & 1.0892 \\
\hline 1 & 1 & 1 & 1 & 1 & 1 & 1 & 1 & 1 & 1 & 1 \\
\hline
\end{tabular}

Figure 5: Temperature distribution for $\mathrm{P}=7$ 


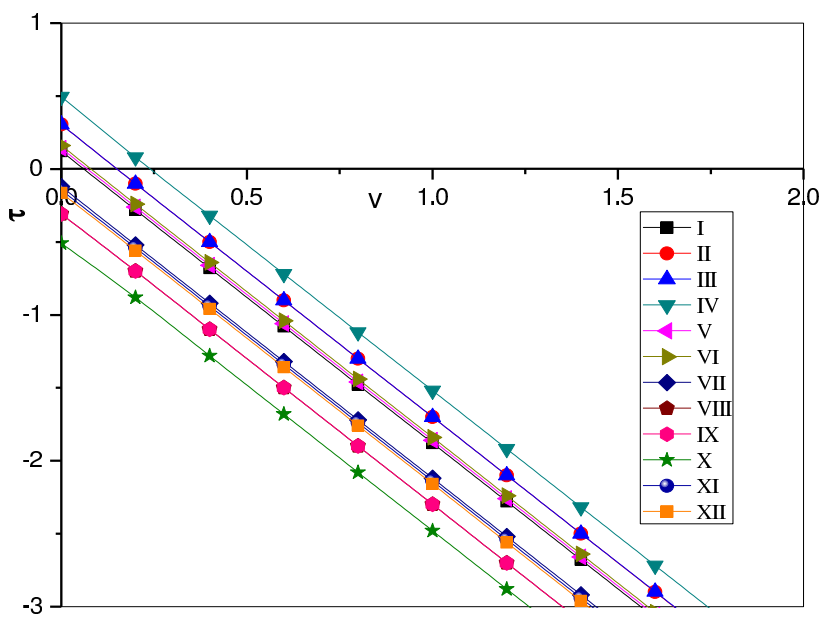

\begin{tabular}{|c|c|c|c|c|c|c|c|c|c|c|c|c|}
\hline $\mathrm{V}$ & $\mathrm{I}$ & $\mathrm{II}$ & $\mathrm{III}$ & $\mathrm{IV}$ & $\mathrm{V}$ & $\mathrm{VI}$ & $\mathrm{VII}$ & $\mathrm{VIII}$ & $\mathrm{IX}$ & $\mathrm{x}$ & $\mathrm{XI}$ & $\mathrm{XII}$ \\
\hline 0 & 0.12186 & 0.30463 & 0.30449 & 0.49439 & 0.14204 & 0.16211 & -0.12 & -0.30927 & -0.30898 & -0.5088 & -0.14408 & -0.16423 \\
\hline 0.2 & -0.28 & -0.1 & -0.1 & 0.08003 & -0.26 & -0.24001 & -0.51998 & -0.69998 & -0.69996 & -0.88 & -0.53998 & -0.55998 \\
\hline 0.4 & -0.68 & -0.50003 & -0.50005 & -0.32 & -0.66002 & -0.64002 & -0.91993 & -1.09987 & -1.09973 & -1.2798 & -0.93993 & -0.95994 \\
\hline 0.6 & -1.08 & -0.9 & -0.90001 & -0.72 & -1.06001 & -1.04001 & -1.3199 & -1.4998 & -1.49959 & -1.6797 & -1.33991 & -1.35991 \\
\hline 0.8 & -1.48 & -1.29994 & -1.29987 & -1.1199 & -1.45998 & -1.43998 & -1.7199 & -1.89978 & -1.89955 & -2.0797 & -1.7399 & -1.7599 \\
\hline 1 & -1.8799 & -1.69982 & -1.69964 & -1.5197 & -1.85993 & -1.83993 & -2.11991 & -2.2998 & -2.2996 & -2.4798 & -2.13991 & -2.15991 \\
\hline 1.2 & -2.2799 & -2.09966 & -2.09931 & -1.9194 & -2.25987 & -2.23987 & -2.51993 & -2.69987 & -2.69975 & -2.8799 & -2.53994 & -2.55994 \\
\hline 1.4 & -2.6798 & -2.49945 & -2.49889 & -2.3191 & -2.65978 & -2.63978 & -2.91998 & -3.1 & -3.09999 & -3.2801 & -2.93998 & -2.95999 \\
\hline 1.6 & -3.0797 & -2.89919 & -2.89838 & -2.7187 & -3.05968 & -3.03968 & -3.32005 & -3.50016 & -3.50033 & -3.6803 & -3.34005 & -3.36005 \\
\hline 1.8 & -3.4796 & -3.29888 & -3.29777 & -3.1182 & -3.45955 & -3.43955 & -3.72013 & -3.90038 & -3.90076 & -4.0807 & -3.74014 & -3.76014 \\
\hline 2 & -3.8794 & -3.69853 & -3.69706 & -3.5176 & -3.85941 & -3.83941 & -4.12024 & -4.30064 & -4.30128 & -4.4811 & -4.14024 & -4.16024 \\
\hline
\end{tabular}

Figure 6: Skin friction at the plates $\mathrm{y}=0$ and $\mathrm{y}=1$ : $\mathrm{P}=0.71$ 


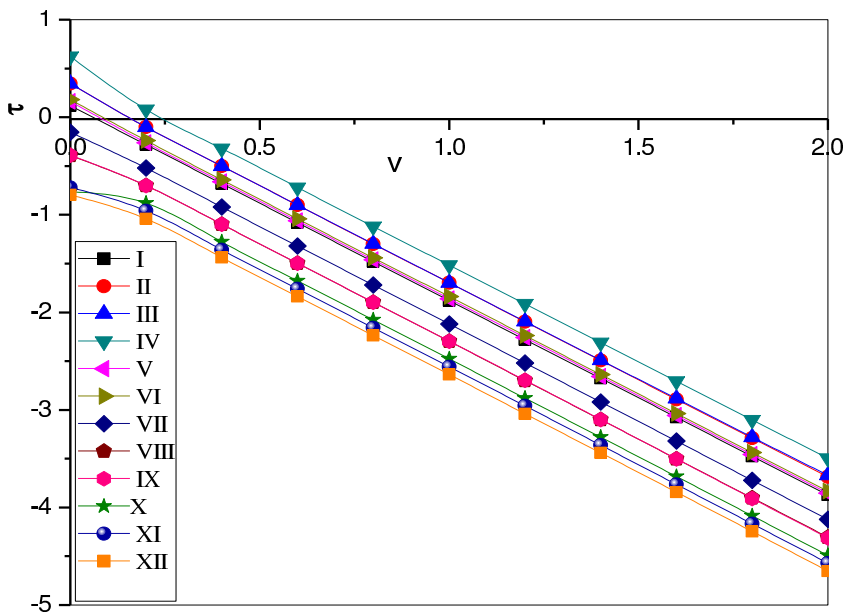

\begin{tabular}{|c|c|c|c|c|c|c|c|c|c|c|c|c|}
\hline $\mathrm{V}$ & $\mathrm{I}$ & $\mathrm{II}$ & III & IV & V & VI & VII & VIII & IX & X & XI & XII \\
\hline 0 & 0.12 & 0.34566 & 0.34426 & 0.62162 & 0.16012 & 0.18083 & -0.15449 & -0.39129 & -0.38846 & -0.7631 & -0.72019 & -0.79473 \\
\hline 0.2 & -0.2801 & -0.1 & -0.1 & 0.08027 & -0.26005 & -0.24005 & -0.5198 & -0.69981 & -0.69963 & -0.8802 & -0.96048 & -1.04073 \\
\hline 0.4 & -0.6802 & -0.50025 & -0.50051 & -0.3202 & -0.66015 & -0.64014 & -0.91934 & -1.09867 & -1.09735 & -1.2784 & -1.35869 & -1.43895 \\
\hline 0.6 & -1.0801 & -0.90004 & -0.90008 & -0.7199 & -1.06006 & -1.04006 & -1.31906 & -1.498 & -1.496 & -1.6774 & -1.75764 & -1.83791 \\
\hline 0.8 & -1.4798 & -1.29937 & -1.29873 & -1.1188 & -1.45978 & -1.43978 & -1.71897 & -1.89779 & -1.89558 & -2.0771 & -2.15734 & -2.23762 \\
\hline 1 & -1.8793 & -1.69822 & -1.69645 & -1.517 & -1.85932 & -1.83932 & -2.11907 & -2.29805 & -2.2961 & -2.4775 & -2.55778 & -2.63807 \\
\hline 1.2 & -2.2787 & -2.09662 & -2.09323 & -1.9144 & -2.25867 & -2.23867 & -2.51935 & -2.69877 & -2.69754 & -2.8787 & -2.95897 & -3.03927 \\
\hline 1.4 & -2.6778 & -2.49454 & -2.48909 & -2.3111 & -2.65784 & -2.63784 & -2.91982 & -3.09996 & -3.09991 & -3.2806 & -3.3609 & -3.44121 \\
\hline 1.6 & -3.0768 & -2.892 & -2.88401 & -2.7071 & -3.05682 & -3.03682 & -3.32048 & -3.50161 & -3.50322 & -3.6833 & -3.76357 & -3.84389 \\
\hline 1.8 & -3.4756 & -3.28899 & -3.27801 & -3.1023 & -3.45561 & -3.43561 & -3.72132 & -3.90373 & -3.90745 & -4.0867 & -4.16699 & -4.24732 \\
\hline 2 & -3.8742 & -3.68552 & -3.67107 & -3.4968 & -3.85421 & -3.83421 & -4.12235 & -4.30632 & -4.31261 & -4.4908 & -4.57116 & -4.65149 \\
\hline
\end{tabular}

Figure 7: Skin friction at the plates $y=0$ and $y=1: P=7$ 


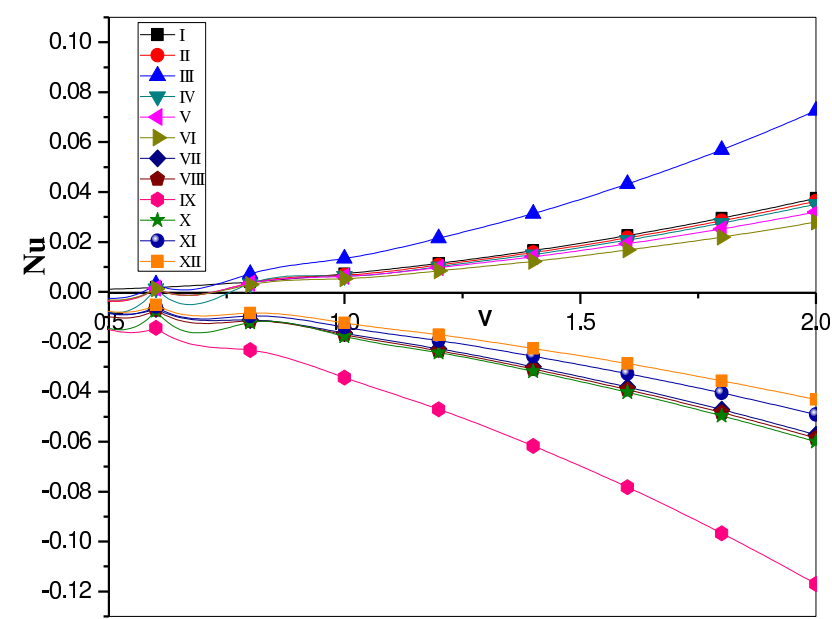

Figure 8: Rate of heat transfer coefficient at the plates $\mathrm{y}=0$ and $\mathrm{y}=1$, $\mathrm{P}=0.71$ 


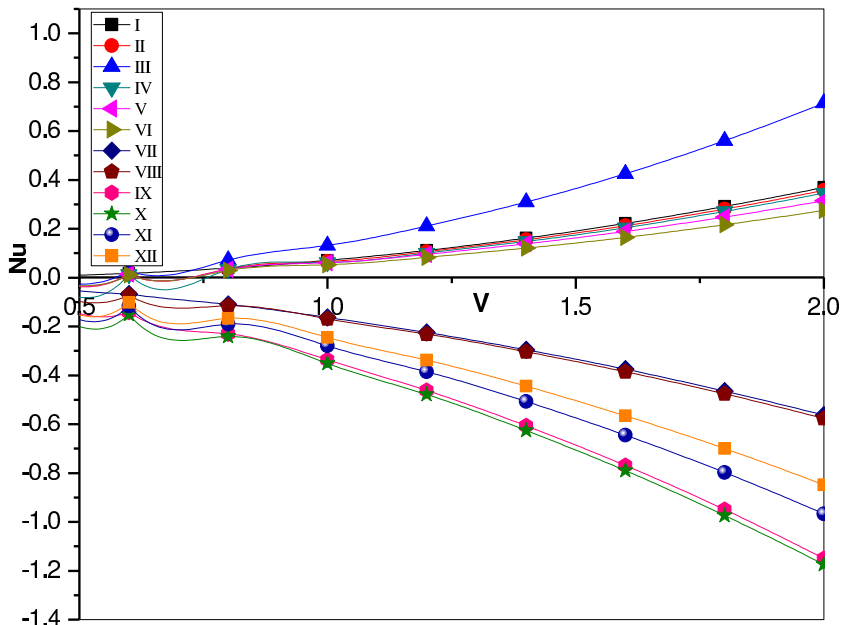

Figure 9: Rate of heat transfer coefficient at the plates $y=0$ and $y=1$ :

$$
\mathrm{P}=7
$$$$
-1.4
$$

\begin{tabular}{|c|c|c|c|c|c|c|c|c|c|c|c|c|}
\hline $\mathrm{V}$ & $\mathrm{I}$ & II & III & IV & V & VI & VII & VIII & IX & X & XI & XII \\
\hline 0 & $1.39 \mathrm{E}-04$ & 0.45719 & 0.44378 & 0.88669 & 0.43128 & 0.3906 & 0.04612 & 0.45548 & 0.44044 & 0.87839 & 0.86131 & 0.78 \\
\hline 0.2 & $1.39 \mathrm{E}-04$ & $8.67 \mathrm{E}-04$ & $1.72 \mathrm{E}-03$ & $2.21 \mathrm{E}-03$ & $1.63 \mathrm{E}-04$ & $1.86 \mathrm{E}-04$ & $-9.47 \mathrm{E}-03$ & -0.01022 & -0.02047 & -0.0232 & $-1.63 \mathrm{E}-02$ & $-1.44 \mathrm{E}-02$ \\
\hline 0.4 & $3.87 \mathrm{E}-03$ & $3.21 \mathrm{E}-03$ & $6.42 \mathrm{E}-03$ & $3.17 \mathrm{E}-03$ & $3.23 \mathrm{E}-03$ & $2.75 \mathrm{E}-03$ & -0.03373 & -0.03588 & -0.07178 & -0.0774 & -0.05815 & -0.05121 \\
\hline 0.6 & 0.01693 & 0.01487 & 0.02975 & 0.01345 & 0.01429 & 0.01232 & -0.0673 & -0.07084 & -0.14163 & -0.1501 & -0.11594 & -0.10201 \\
\hline 0.8 & 0.03932 & 0.03586 & 0.0717 & 0.03306 & 0.03335 & 0.02887 & -0.1102 & -0.11511 & -0.23001 & -0.2413 & -0.18968 & -0.16677 \\
\hline 1 & 0.07103 & 0.06617 & 0.13226 & 0.06198 & 0.0604 & 0.05243 & -0.16242 & -0.16867 & -0.33693 & -0.3509 & -0.27939 & -0.2455 \\
\hline 1.2 & 0.11208 & 0.1058 & 0.21145 & 0.10023 & 0.09545 & 0.08298 & -0.22396 & -0.23154 & -0.4624 & -0.4789 & -0.38505 & -0.33819 \\
\hline 1.4 & 0.16245 & 0.15475 & 0.30926 & 0.1478 & 0.13849 & 0.12052 & -0.29482 & -0.3037 & -0.6064 & -0.6253 & -0.50668 & -0.44485 \\
\hline 1.6 & 0.22214 & 0.21302 & 0.42568 & 0.20468 & 0.18952 & 0.16506 & -0.375 & -0.38517 & -0.76894 & -0.7902 & -0.64427 & -0.56547 \\
\hline 1.8 & 0.29116 & 0.28062 & 0.56073 & 0.27089 & 0.24855 & 0.2166 & -0.46451 & -0.47594 & -0.95002 & -0.9736 & -0.79782 & -0.70006 \\
\hline 2 & 0.36951 & 0.35754 & 0.7144 & 0.34642 & 0.31558 & 0.27513 & -0.56334 & -0.57601 & -1.14964 & -1.1753 & -0.96733 & -0.84862 \\
\hline
\end{tabular}




\section{References}

[1] A. Ogulu, E. Amos, Asymptotic approximations for the flow field in a free convective flow of a non-Newtonian fluid past a vertical porus plate, International Communications in Heat and Mass Transfer, 32 (2005), 974982.

[2] J. Prathapkumar, J.C. Umavathi, I. Pop, M. Basavaaj, Biradar, Fully developed mixed convection flow in a vertical channel containing porous and fluid layer with isothermalor isoflux boundaries, Transport Porous Media, 80 (2009), 117-135.

[3] K.R. Rajagopal, A.S. Gupta, An exact solution for the flow of a nonNewtonian fluid past an infinite porous plate, Meccanica, 19 (1984), 1948 -1954 .

[4] B.M. Rajasekhara, N. Rudraiah, B.K. RamaiahCouette, Flow over a naturally permeable bed, Journal of Maht. Phy. Sci., IIT Madras, 9 (1975), 49.

[5] N. Rudraiah, R. Veerabhadraiah, Temperature distribution in couette flow past a permeable bed, Proceedings Mathematical Sciences, 86 (1977), 537547.

[6] S. Sreenadh, P.V. Arunachalam, A model for the study of flow between two permeable beds, In: Proceedings of the National Symposium on Mathematical Modeling, Mehta Research Institute, Allahabad (1982).

[7] F.J. Suriano, K.T. Yang, J.A. Donlon, International Journal of Heat and Mass Transfer, 8 (1965), 815-831.

[8] J.C. Umavathi, M. Shekar, Unsteady mixed convective flow and heat transfer in a vertical corrugated channel with composite porous media, Journal of Engineering Physics and Thermophysics, 86 (2013), 754-765.

[9] K. Vajravelu, Free convection heat transfer between two long, vertical, thin plates moving in opposite directions, Indian Journal Of Tecnology, 16 (1978), 399-403.

[10] K. Vajravelu, S. Sreenadh, P. Lakshminarayana, The influence of heat transfer on peristaltictransport of Jeffrey fluid in a vertical porous stratum, Commun Nonlinear SciNumerSimulat, 16 (2011), 3107-3125. 
[11] K. Yamamoto, Z. Yoshida, Flow through a porous wall with convective acceleration, J. Phys. Sot., Japan, 37 (1974), 774-779. 